
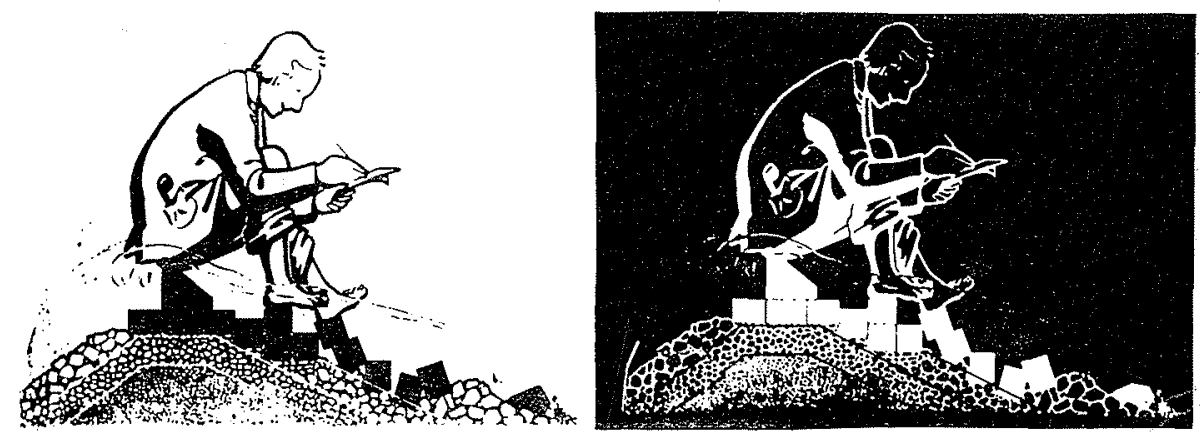

\title{
A research \\ facility with concurrent air and water flows
}

\author{
BY \\ E. J. PLATE *
}

\section{Introduction}

The Fluid Mechanics Program at Colorado State University has been augmented recently by the addition of a new laboratory flume in the University's Fluid Dynamics and Diffusion Laboratory. This facility enables simultaneous control of the open-channel flow and the air flow.

The facility will be utilized for research involving the study of wind and water interactions. Problems that can be investigated are associated with the wind drag on open-channel flow, the generation and development of water surface waves on standing and moving water, and the effect of wind on transport and diffusion processes.

These problems bridge two research areas of Colorado State University's Engineering Research Center, namely the Hydraulics and Fluid Mechanics Programs.

The facility

The wind-water channel was designed with applications to open-channel flow in mind. There-

* Associate Professor of Civil Engineering, Fluid Dynamics and Diffusion Laboratory, Colorado State University, Fort Collins, Colorado, U.S.A. fore, a length of 44 feet was chosen so that a uniform depth could be established for slopes up to five percent. The length is, however, not sufficient to obtain a fully developed wave pattern which does not change with increase in fetch length.

The maximum design depth for water flow is six inches, with a free board of two inches added on each end of the facility. At this depth, and with the facility at maximum slope, a Froude number of about four can be obtained for the open-channel flow.

Uniform depth in the channel is obtained by tilting the facility about a pivot which is located approximately under the one-third point of the test section as shown in Figure 1 and 2. The structure is kept statically determinate by providing a rotating support at one end of the facility. The support column rests on wheels riding on two tracks. A drive screw passes through a two inch nut which is fastened to the axis of the wheels. The drive screw is powered by a $1 / 2 \mathrm{HP}$, gear reduced and reversible ac-motor which permits convenient slope adjustment. The slope is indicated through the voltage across a potentiometer which is driven by the motion of the wheel axis.

The test section is protected from vibrations at all areas of contact with vibration isolation sections. A rubber joint with U-shaped cross section is installed between the test section and the fan. Also, the inlet of the test section is connected to the pump through a sixteen inch rubber hose in the vertical pipe section of the supply pipe, as 


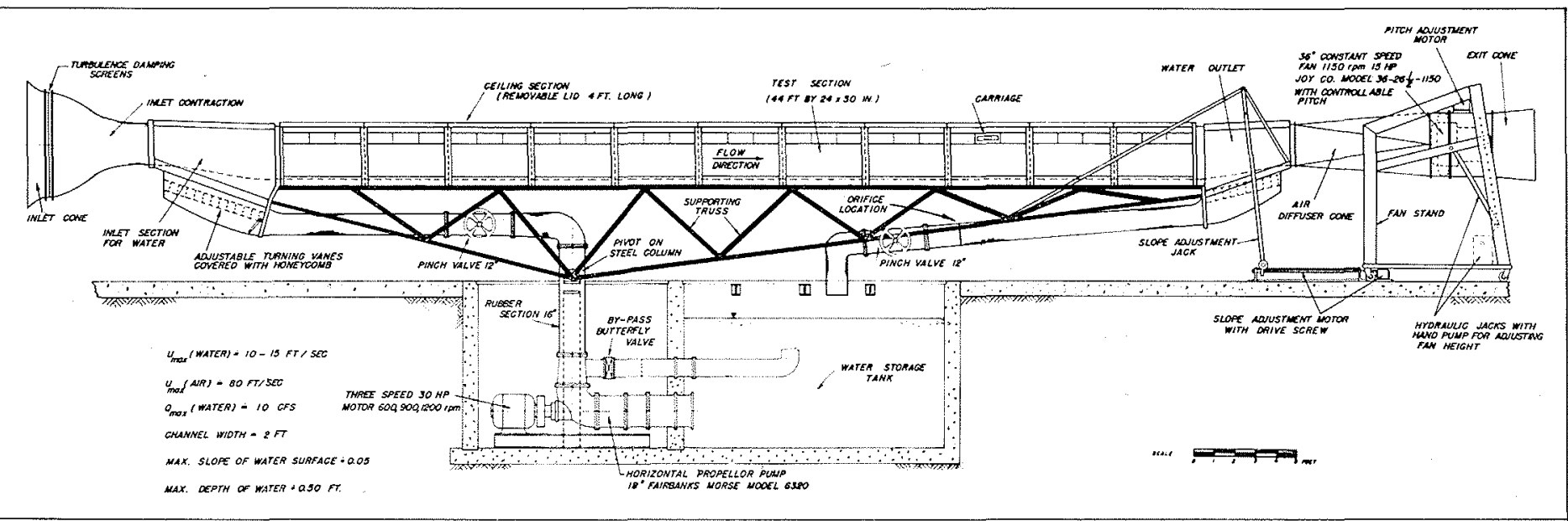

General view of wind water facility

II) Schéma général de l'installation expérimentale

shown in Figure 1. The hose not only effectively prevents vibrations from being transmitted from the pump through the pipe but also dampens out small pressure fluctuations in the water which could produce vibrations. A third area of potential vibration transmission is from the pump through the sump walls and the pivots. This was avoided by supporting the pivots on steel columns which stand on foundations separated from that of the pump.

The water circulation is maintained through an eighteen inch horizontal propellor pump which is equipped with a three-speed $30 \mathrm{HP}$ motor. The discharge is coarsely controlled by a ten-inch by- pass equipped with a butterfly valve. The by-pass serves also to avoid unstable pump operation associated with low discharges. The discharge is finely controlled by the inlet valve. The valves of the inlet and outlet are rubber pinch valves. They were chosen because they seemed to offer the advantage of low separation-induced vibrations at reasonable cost.

The water inlet and outlet sections must produce smooth water surface conditions without any protrusions like gates extending into the air flow. Therefore, the water enters and leaves the test section through turning vanes in the bottom of the inlet and outlet section on which honeycomb

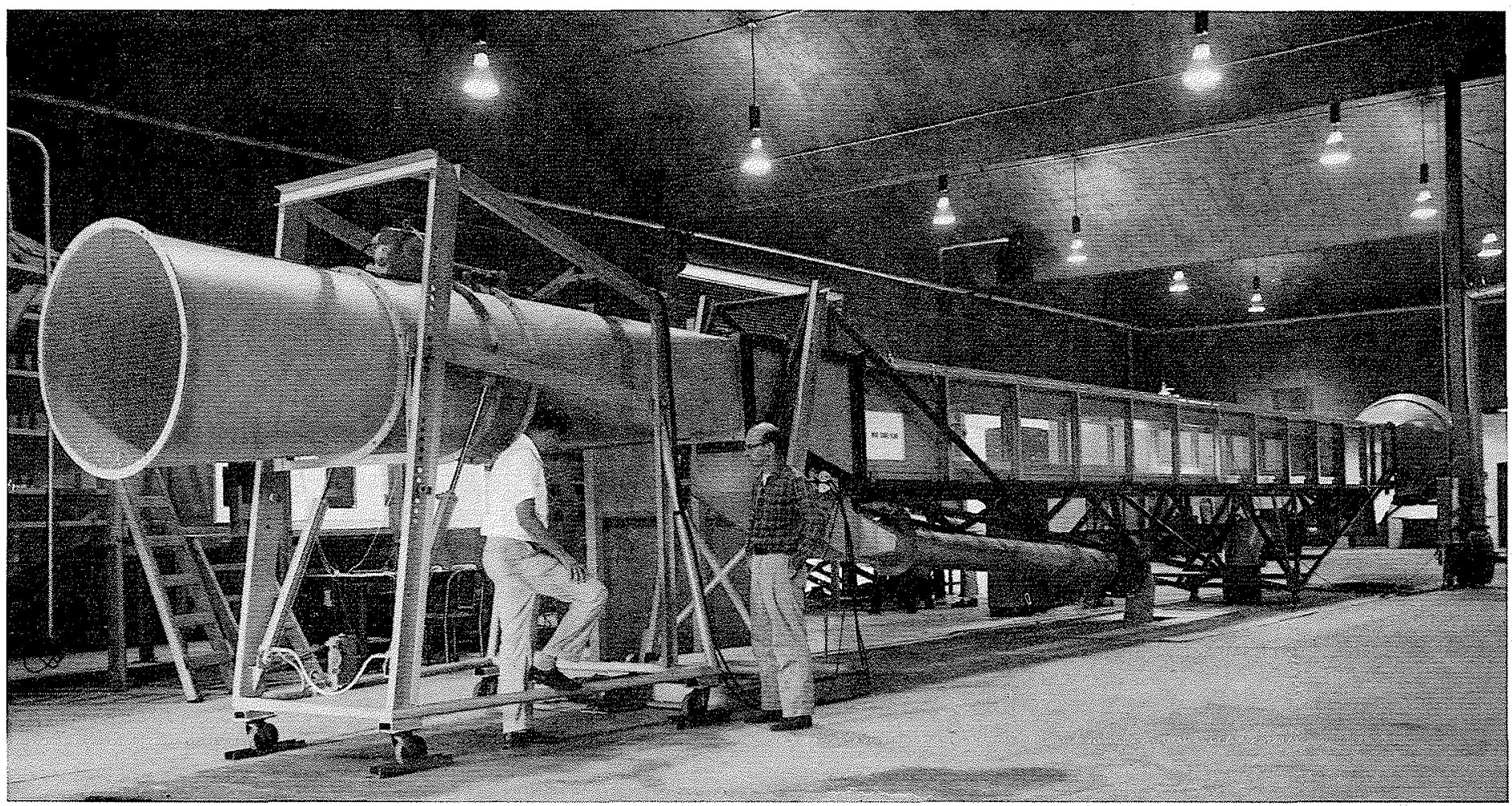

The wind water facility 
straighteners are placed. A heavy aluminum honeycomb of six inch thickness and cell openings of $3 / 8$ inch lies on top of the vanes. Over this honeycomb two thin sheets of honeycomb of $1-1 / 2$ inch thickness and $1 / 4$ inch cell diameter were placed on support brackets. The thin sheets were shaped into a transition.

The honeycomb transitions serve three purposes. They help to spread out the entering water over a wide area, so that it enters at a low velocity and therefore the water surface changes are small. For improving the water distribution at the inlet, the turning vanes can be adjusted by a lever, and the best configuration of the entering water can be set by trial and error. The honeycomb surface also provides a transition for the air flow when the water surface is at low elevations. Finally, the honeycomb cell structure of the inlet and outlet provides a beach which prevents reflection of waves traveling on the water surface.

Connected to the outlet is a sixteen foot length of twelve inch pipe with a pinch valve serving as tail gate. An orifice is built into the pipe two feet upstream from the valve. The orifice is calibrated in place by removing the whole pipe and placing it into the calibration stand of CSU's hydraulic laboratory. From the pipe, the water is returned to the sump.

The air flow is controlled by a thirty-six inch commercial fan which is directly connected to a $15 \mathrm{HP}$ induction motor. Wind speeds of from 0 to $80 \mathrm{fps}$ can be obtained by remotely adjusting the pitch of the fan blades. The air is taken in from the laboratory building into the entrance section. The entrance section has a bell-shaped circular inlet, two screens and a contraction cone where the air is accelerated and shaped into a jet with rectangular cross section. The contraction cone was molded in fiberglass.

Downstream from the test section the air flow is led through a honeycomb which eliminates asymmetry of the velocity distribution of the air. The air passes into the fan through a diffuser in which the duct cross section is changed from square to round. The air is discharged through an outlet cone into the laboratory building.

When the slope of the test section is changed, the fan elevation and inclination has to be changed also. Therefore, the fan is supported on a system of levers which can be actuated by hydraulic jacks. The fan support rests on casters which permit movement of the fan during rotation of the test section, or to the other end of the test section when the direction of air flow is to be reversed.

Instrumentation

The facility is instrumented for taking profiles of the mean velocity and the turbulent intensity of the air, as well as profiles of mean velocity in the water, and the elevation of the instantaneous water surface as function of time and distance from the test-section entrance. The data acquisition system and the methods of data handling are illustrated schematically in Figure 3.

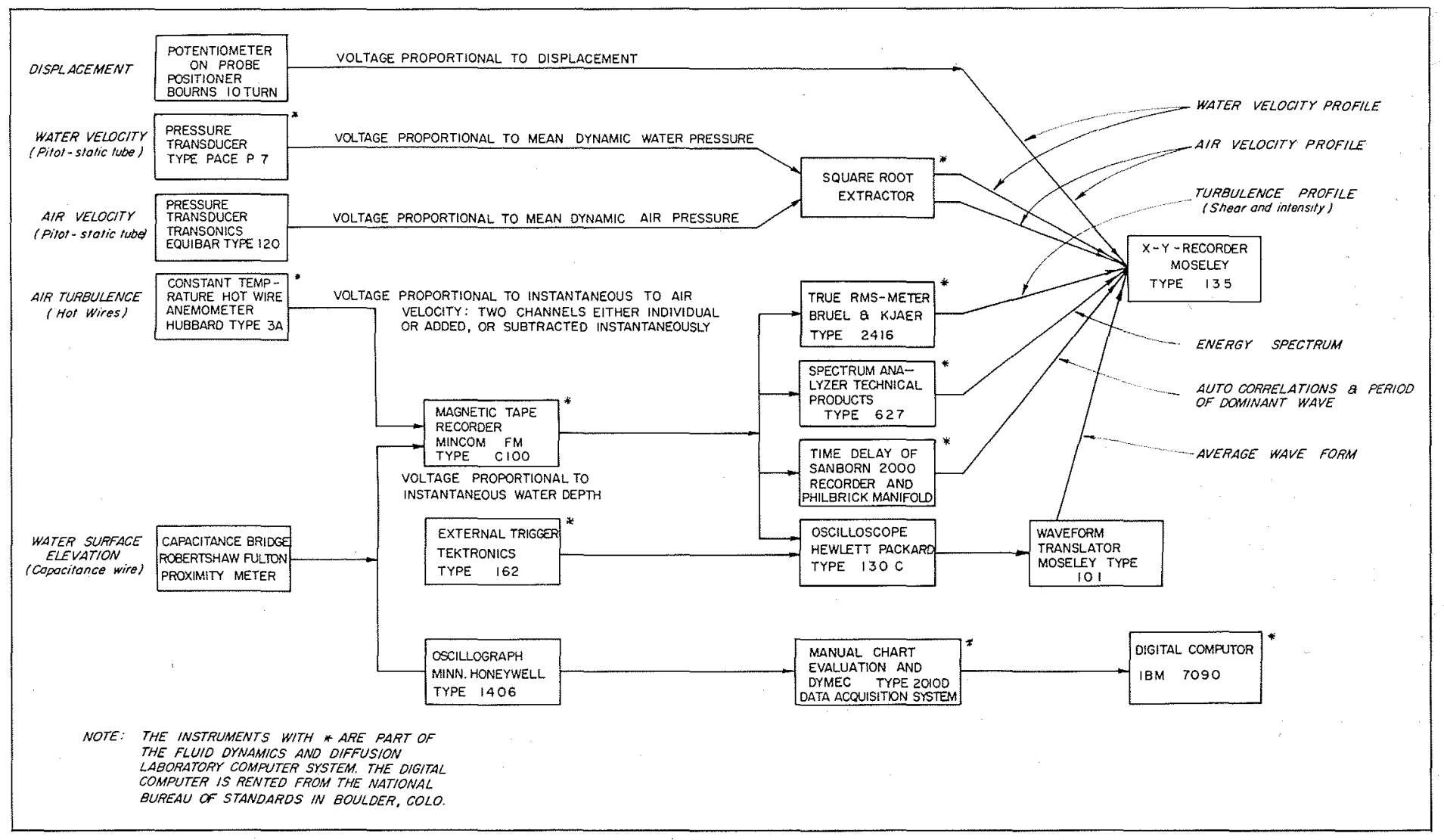




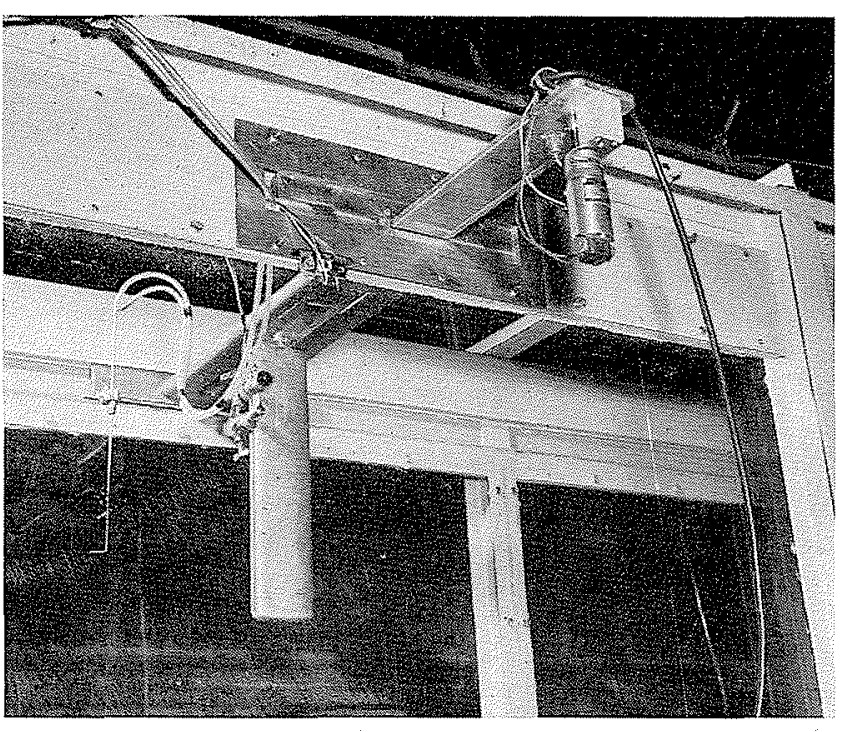

4) The probe positioner.

Dispositif de mise en position de la sonde de mesure.

Velocity-profile data are taken with a probe positioner shown in Figure 4. A vertical, air-foil shaped beam of large stiffness contains a screw on which the probe travels up and down. The motion of the probe is sensed by a multiturn potentiometer which is used for obtaining a voltage proportional to probe distance from the test-section floor. The probes are pitot tubes for mean velocity flow profiles, and hot-wire anemometers for measurements of time varying velocity components. A complete set of analog computing equipment is available to perform a statistical analysis of the turbulent quantities which is illustrated in Figure 3. Profiles of time-mean quantities are plotted directly versus distance on an $\mathrm{x}-\mathrm{y}$ plotter.

A statistical analysis of the time varying water surface can at the present not conveniently be performed with the analog equipment. A program has been initiated to investigate the errors which are introduced if the time of the recording is artificially reduced to a usable computer time by recording and reproducing at different magnetic tape recorder speeds, or perhaps even by re-recording on a second tape recorder. In this manner, the frequencies of the waves can be increased, at least theoretically, until they are compatible with the analog-computer frequency band. At present, however, the analysis is made with digital equipment.

The instrumentation will be extended in the near future for measuring instantaneous water and air velocities at known instantaneous distances from the water surface. Also, it is planned to control the temperature of the air and the water at the test-selection inlet. Instruments for measuring mean distributions of temperature in air and water are already available.

\section{Acknowledgments}

Financial support for building and instrumenting the described facility was obtained from the National Science Foundation under Grant No. G.-19793. Administrative support was given by Dr. A.R. Chamberlain, Vice President, Colorado State University. 